\title{
Biliary atresia - a new derivative method?
}

\author{
Murar $\mathrm{E}^{1}$, Barta $\mathrm{A}^{2}$, Omanik $\mathrm{P}^{3}$, Trnka $\mathrm{J}^{3}$ \\ Pediatric Surgery Department, Children's University Hospital, Comenius University, Bratislava, \\ Slovakia.pavol.omanik@gmail.com
}

\begin{abstract}
Background: Biliary atresia is a progressive cholangiopathy in neonates of unknown origin. Surgical intervention - Kasai portoenterostomy - is the only treatment possible. However, only liver transplantation can be considered a definitive solution even in cases of favourable post-operative course, i.e., after bile passage has been recreated.

Objectives: The authors set out to identify ways to optimize the operative treatment in biliary atresia patients. In particular, their objective was to identify a suitable bile derivation area outside porta hepatis, thereby simplifying the surgical procedure of the following liver transplantation.

Methods: The research was conducted from 2006 to 2010 on a set of 30 corrosive casts prepared by the Institute of Anatomy, Medical Faculty, Comenius University Bratislava. The research consisted of an analysis of individual tubular structures of the liver parenchyma, particularly the bile vessels. The authors explored the latter's position and branching out, as well as their relationships in different segments, and their availability in surgical exploration.

Results: The analysis of the corrosive casts resulted in selecting an area in liver segments II and III suitable for creating a new type of hepatoenterostomy. The area is sufficiently distant from porta hepatis, conveniently accessible to the surgeon, and has a broad bile duct branch. This type of anastomosis would preserve the anatomic situation in the porta hepatis area intact for the following transplantation.

Conclusion: While the results of the experiment warrant some optimism, authors will have to wait until this type of derivation operation is applicable in practice. The authors continue their research by performing anastomosis in an animal model (Fig. 5, Ref. 18). Text in PDF www.elis.sk. Key words: biliary atresia, corrosive cast, hepatoenterostomy.
\end{abstract}

Biliary atresia (BA) is a life-threatening idiopathic neonatal progressive cholangiopathy in nurslings. With incidence of approximately 1:15000 live births, it is among the most frequent congenital liver diseases (1). Up to 90 percent of patients die before reaching the age of 3 years without an early surgery (1). Due to the occurrence of irreversible changes in the liver parenchyma, differential diagnosis should be made within 4-6 weeks (2). Patients with confirmed diagnosis undergo, first and foremost, a drainage operation; Kasai portoenterostomy has become the golden standard over the years. During the operation, extensive dissection of fibrous residues of extrahepatal bile ducts in the porta hepatis takes place, after which the small intestine is anastomosed to the dissected "crater" in porta hepatis area, to create a portoenterostomy. Regular bile ducts are developed in the porta hepatis in about $10-20 \%$ of BA patients, allowing the surgeons to perform even hepaticoenterostomy $(3,4)$. Unfortunately, the quality of life of

${ }^{1}$ Pediatric Surgery Department, Children's University Hospital, Comenius University, Bratislava, Slovakia, ${ }^{2}$ The Institute of Normal and Pathological Physiology, Slovak Academy of Sciences, Bratislava, Slovakia, and ${ }^{3}$ Pediatric Surgery Department, Children's University Hospital, Medical Faculty, Comenius University Bratislava, Slovakia

Address for correspondence: P. Omanik, MD, PhD, Pediatric Surgery Department, Children's University Hospital, Comenius University, Limbova 1, SK-833 40 Bratislava, Slovakia.

Phone: +421.2.5937 1307
BA patients remains significantly affected despite successful surgical intervention. The condition further proceeds as the patient is put on the waiting list for liver transplantation, which is the final surgical step in BA treatment (5-7).

The authors have attempted to locate a new target area for hepatoenterostomy in the liver parenchyma, which would leave the porta hepatis area intact and therefore in a better condition for the transplantation later on.

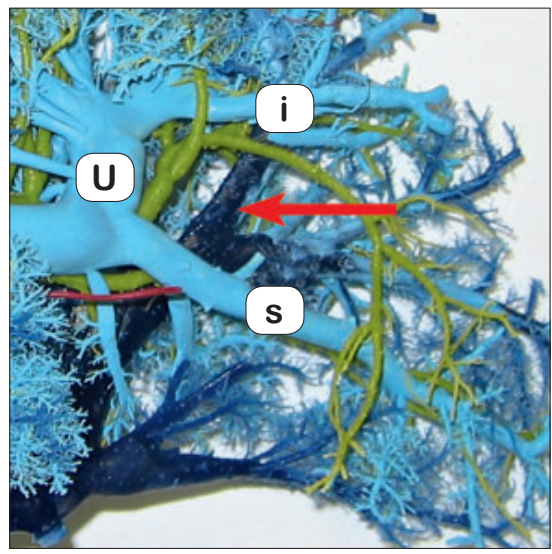

Fig. 1. The "crayfish pincher" (U - pars umbilicus of the left portal vein branch, $i$ - lateroinferior branch of portal vein, $s$ - laterosuperior branch of portal vein, arrow - left hepatic vein branch). 

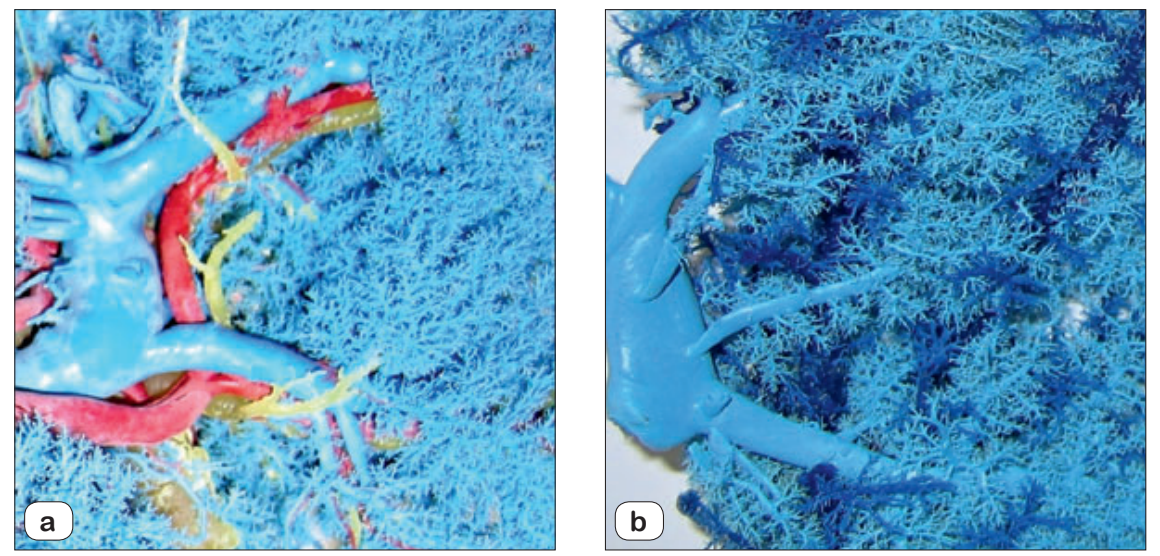

Fig. 2. Variants of the portal vein course (variants $A$ and $B$ - read the article for details).
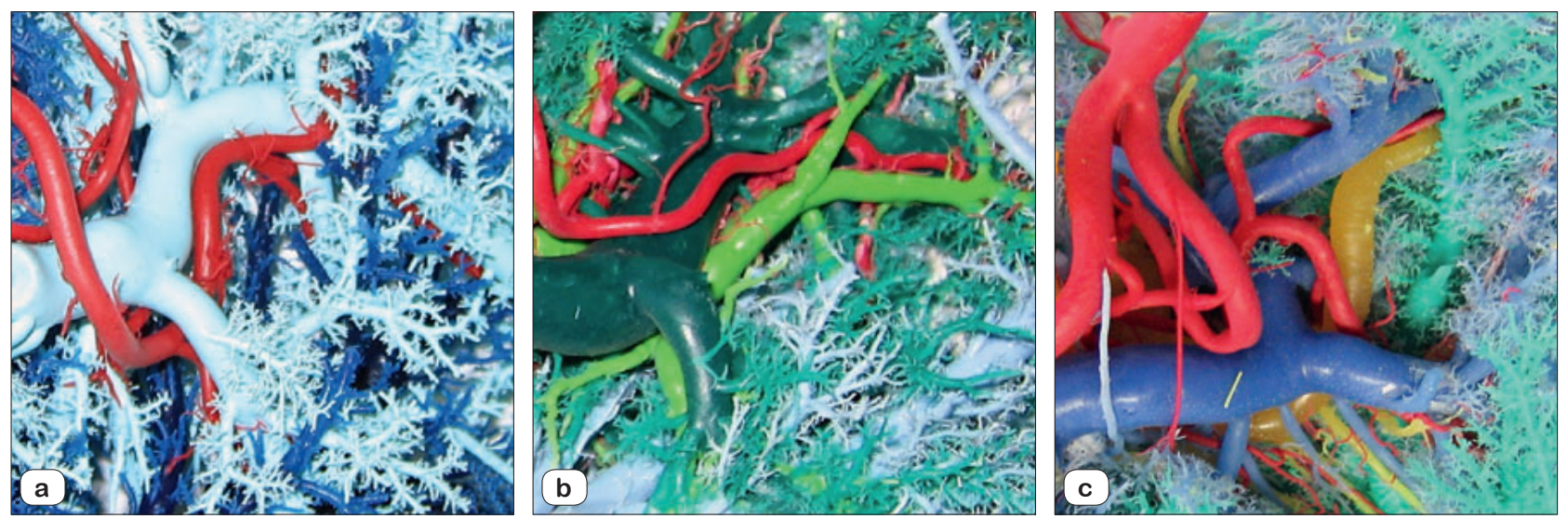

Fig. 3. Variants of the hepatic artery course (variants A, B and C - read the article for details).
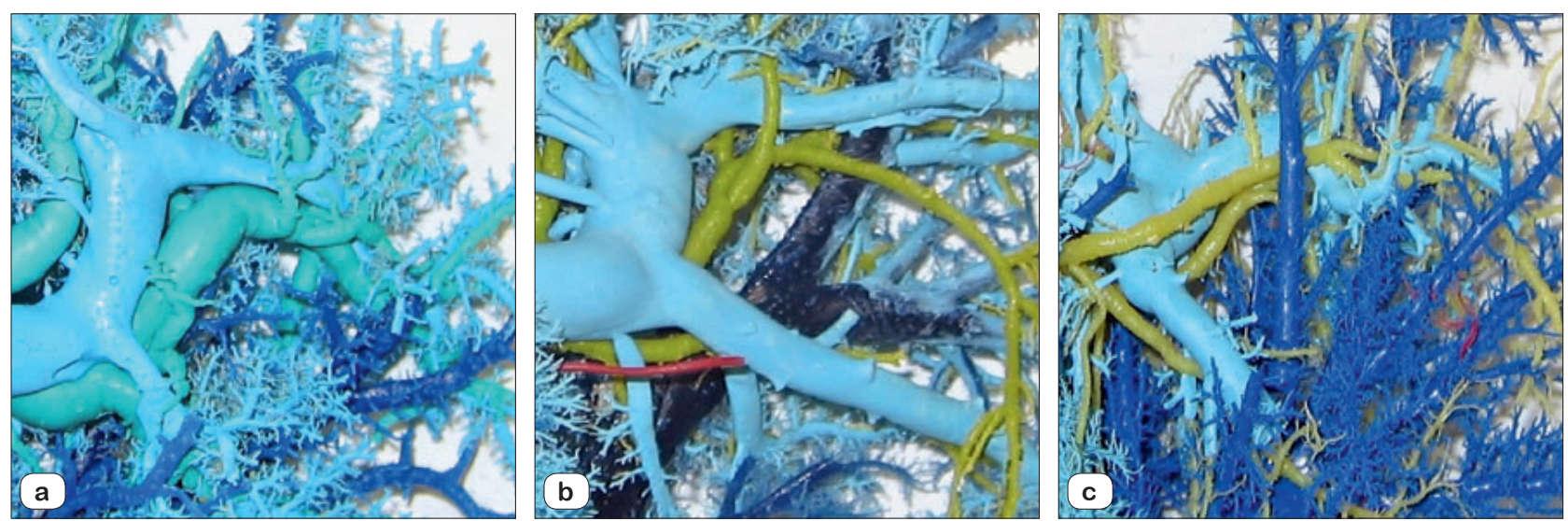

Fig. 4. Variants of the lateroinferior branch of hepatic duct course (variants A, B and C - read the article for details).

\section{Methods}

The research was conducted from 2006 to 2010 on 30 corrosive casts of the liver prepared in the Institute of Anatomy, Medical Faculty of Comenius University in Bratislava. The livers used for the casts were cadaverous organs obtained within 48 hours after death of adult subjects without a previous history of liver disease, whose liver looked macroscopically intact during autopsy. The organ itself was taken from the donor with sufficiently long blood vessels and bile ducts in the porta hepatis and retrohepatally. After individual vessels and the common bile duct were made accessible and cannulated with polyethylene cannules, the vessel system was first washed with saline solution. The two-component acrylate Duracryl Plus, colour code O, and Spofakryl (Spofa Dental, Prague) suitably 


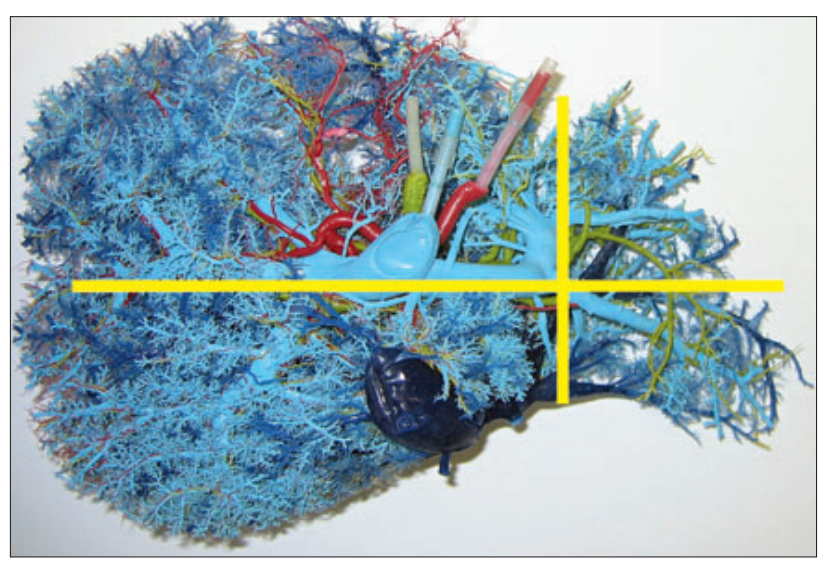

Fig. 5. Corrosive liver cast (vertical line - falciform ligament course, horizontal line - imaginary prolongation of the course of the pars transversus of left portal vein. The intersection marks the bottom edge of $\mathrm{DZ}$ - read the article for details).

hued with a pigment (Batson's pigments, Polysciences Inc.) was prepared. The proper hepatic artery was subsequently instilated with a red acrylic mixture, the portal vein was instilated with blue one and the common bile duct with green one. The whole organ was then placed in a tepid water bath to allow for polymerization within approximately 30 minutes. Following intraluminal hardening of the acrylate, the parenchyma was placed in a hot bath of 2 $\%$ potassium hydroxide for 3-4 hours until the full corrosion of the tissue. In the next step, the hardened cast of vessels was cooled with water and subjected to investigation. The topographic and metric investigation consisted from the observation of the course of and interrelation among the tubular structures, specifically bile ducts, in the liver parenchyma.

\section{Results}

The proper research on the corrosive casts was preceded by a speculation about an area outside of the porta hepatis, where an alternative drainage operation could occur. Such an area should satisfy the criteria of sufficient distance from the porta, presence of a broad bile duct branch, and finally good accessibility for the surgical procedure. Based on liver anatomy, we assume that the left liver lobe (segments II and III) could be potentially the best area to create a hepatoenterostomy of the new type. Therefore, the following analyses focus on this area.

\section{Organization of anatomic structures in the derivation area}

The analysis of the corrosive casts has corroborated a variability in the course of all tubular structures, i.e., bile ducts, arteries and veins. Our identification of an area where the course of the branch of portal vein, artery and bile duct was identical or showed minimal anatomic variation in all 30 casts has been an important conclusion. We will refer to this spot as the derivation area (DA) hereinafter. The area where surgical preparation of bile ducts could be performed and a hepatojejunostomy constructed will be referred to as the derivation zone (DZ).
The derivation area (DA) is created by the triad of the tubular structures in the following order (as seen by a surgeon observing the visceral surface): first a portal vein branch, so-called pars umbilicus (8), seconds a hepatic artery branch, and third a hepatic duct branch (vein, artery, duct - VAD). The triad runs in parallel with the falciform ligament (FL). In an in-vivo liver, VAD is divided by FL; the pars umbilicus is always to the left and the artery and bile duct to the right (peroperative view to visceral surface) (Fig. 1).

The derivation zone (DZ) borders on the pars umbilicus belonging to the left portal vein branch medially, on the lateroinferior branch of portal vein in the ventral direction, and on the laterosuperior branch of portal vein in the dorsal direction (8). There is no precise demarcation on the lateral part of the zone; its distance from FL (pars umbilicus of portal vein) varies individually. (Fig. 1).

\section{Analysis of the course of anatomic structures in the derivation area}

The central view of DA remained stable during our analysis of the corrosive casts; the order in which the VAD structures run next to one another was identical in all cases. However, differences can be observed in their interrelations, such as lumens of individual structures or the places where they cross FL to enter lateral segments of the liver. The artery branch manifests the highest variability, followed by the portal vein branch and the bile duct branch. Variation in branches of the left hepatic vein in the area were not analyzed in detail as these do not play a role in the operation. The branches ran consistently deeper and more laterally in the liver parenchyma where surgical exploration will not be necessary.

The branch of portal vein, pars umbilicus, ran in parallel to and medially from FL. Two branches run from the pars umbilicus to the lateral segment: lateroinferior branch for segment III and laterosuperior branch for segment II, looking the semblance of a "crayfish pincher" (Fig. 1). This classic shape was uniformly observed in all the corrosive casts. In 8 cases $(27 \%)$, smaller portal vein branches entered the lateral segment through FL together with the above branches. The detachment of these branches was not constant. Even if these smaller branches do not occur in all cases, the surgeon must expect them in DZ (Fig. 2).

The artery branch accompanies the pars umbilicus of portal vein and subsequently most often runs behind the laterosuperior branch of portal vein (variant A). In approximately $1 / 3$ of cases, one should expect that the artery branch will pass above the laterosuperior branch of portal vein (variant B). Only in $7 \%$ of cases did the artery continue to the direction towards lateroinferior branch of portal vein (variant C). What is important, however, is the uniformity of its parallel course with the pars umbilicus of portal vein between the vein and the bile duct (Fig. 3).

The course of the bile ducts was uniform as they entered FL. Variant A was observed in 28 casts out of the total of 30, i.e. in $94 \%$. Only the remaining 6\% showed minor variation (variants B and C) (Fig. 4).

While the above variations in the course of tubular liver structures are known, it is important to remember that exploration in DZ will always open a sufficiently broad bile duct, i.e., the lateroinferior branch of bile bile duct, which transports bile from segment 
III. In dissection from this branch towards FL, the surgeon must expect to encounter a branch of the hepatal artery, i.e., lateroinferior branch. Both the structures will always be located to the right of FL from the surgeon's point of view.

\section{Locating the exact preparation area in a native liver}

Dissecting the liver in DZ, the surgeon obviously lacks the view offered by the corrosive cast. It would help if he could orientate himself using certain structures which are very likely to demarcate the borders of the preparation area. The key structures during in situ preparation include:

1) Portal vein in porta hepatis. The left branch of the portal vein (pars transversus) originated from it, almost always running perpendicularly to FL (8). The right bottom angle of the derivation zone - DZ (Fig. 5) is located where the pars transversus of portal vein crosses FL (X point). Localization of $X$ point is easier if the ligamentum venosum, which runs into the left portal vein, can be dissected.

2) Falciform ligament. Dissection takes place left of FL, ventrally from X point. Analysis of metric parameters confirmed the presence of a bile duct in the middle third of the length of FL; it accounted for approximately $25 \%$ of the FL length (Fig. 5).

3) Branches of the left portal vein - lateroinferior branch and laterosuperior one. In dissection, these demarcate the area of the future anastomosis from the front and from the back. Dissecting these, the surgeon must always expect to encounter a bile duct branch from which bile can be derived. If the surgeon starts dissection at the level of the middle third of FL, he will always find himself in a DM area with lateroinferior branch of bile duct.

In situ explored tubular structures run relatively close to the surface in the parenchyma. The proportion of the average depth of the observed structures below the visceral surface to the average thickness of the liver in the FL area gives a value which represents surgical exploration down to approximately $1 / 4$ of the parenchyma thickness. The left hepatic vein branches should not be reached because of their deeper and more lateral location (Fig. 1).

\section{Discussion}

Kasai portoenterostomy has become the golden standard in BA treatment. Early diagnosis, surgery, and follow-up treatment increase the patients' chances of survival. In the past, BA patients survived a maximum of 2-3 years. The surgical treatment has increased survival several times in early diagnosed patients. The use of portoenterostomy results in a 5-year survival in 30-75\% of patients (2). Prognosis and the patient's age at the time of operation are inversely proportional. Patients who undergo a Kasai procedure within 2 months of life have a longer survival interval than those who are operated later in life ( $83 \%$ vs $35 \%$ ) (2). Therefore, McKieman et al stress that such patients should be managed in surgical centres with at least 5 cases a year (9).

The key success factor in this type of surgical treatment is whether bile passage takes place through the anastomosis in postoperative period. With this in mind, several surgeons attempted to modify the classic Kasai procedure in recent years so as to increase the probability of opening intrahepatal bile ducts. All of these modifications shared a common focus of increasing the lateral dissection in the porta hepatis.

Endo et al emphasize that a regular dissection in the porta hepatis may be increased between and along the right branch of portal vein, and may include the area of potentially usable residues of intrahepatal bile ducts (10).

In studying cadaverous casts, Ito et al found a topographic correlation between intrahepatal bile ducts in BA sufferers and patients with intact livers. They performed an extensive portal exploration to reach the obstructed ends of main intrahepatal bile ducts (11).

Toyosaka et al also achieved good results in a rather extensive dissection using the portal vein as a conduit. These authors provide the fact that bile ducts can also be opened through dissection behind the fibrotic tissue, which does not extend too deep in the liver as a reason for performing an extensive dissection. The common bile duct usually receives bile from the main branches in the porta hepatis, which run upward and medially to turn sideways dorsally in the liver. Dissection on lateral sides, where these branches occur, is therefore very important (12).

Ando et al describe a new technique of fibrotic residues dissection in a suitable direction through splitting the ligamentum venosum; departing from the left portal vein, the ligamentum venosum is ligated and cut where it bifurcates to the lateral segment and the umbilical part, giving a broad view of the porta hepatis. The fibrotic area of the porta hepatis can thus easily be dissected in the dorsal and lateral parts, where multiple bile ducts are present (13).

Schweizer et al point out that bile ducts in porta hepatis area terminate in three definable zones: central and two lateral ones. Schweizer stresses that opening the bile ducts in the central zone by itself does not guarantee sufficient bile drainage. Including the intact bile ducts of the two lateral liver lobes in the anastomosis may improve the prognosis of the Kasai procedure (14).

To verify if anastomosis to the newly proposed derivation area is functional, the anastomosis will have to be performed in an animal model after artificially inducing a BA-equivalent condition. We dare assume that bile derivation will be successful as a sufficiently broad bile duct - lateroinferior branch of left hepatic $d u c t$ - was consistently identified in DA in our analysis of the corrosive casts. Through an opening in it, bile from at least the left segments of the liver would be derived into the gastrointestinal tract. On the contrary, the Kassai procedure often yields debatable derivation results: bile ducts with real lumen may not be present in the porta hepatis $(5,6,15)$.

In patients with such type of BA, when communication between the right and left liver lobes via a regular junction of the right and left hepatic duct is present, unobstructed bile derivation from all liver segments can be assumed through DA. The fibrosis process has been proven to continue after successful bile derivation; similarly, parenchyma regeneration has been proven in the form of large nodules in the porta hepatis area $(16,17)$. Both of the processes could run in the whole liver in the new type of derivation anastomosis.

In patients suffering from the type of BA in which no bile communication between the right and left liver lobes takes place, the 
new type of anastomosis will drain bile from the left lobe only. Such intrahepatal bile ducts links between left and right lobes do not occur in man - unlike some animals (18). Fibrotization and nodular parenchyma regeneration can then be expected in the left lobe only, whereas the right lobe, where bile stasis continues, continues to experience fibrotic changes. However, the legitimacy of such prognoses can only be confirmed in the next, in vivo phase of the experiment using an animal model.

\section{Conclusion}

Many surgeons specializing in hepatobiliary surgery have been looking for ways to improve bile drainage from the liver affected by biliary atresia. They have, however, mostly looked in the porta hepatis area, thus contributing to modifications of the Kasai procedure. This type of operation, however, does not address the problem of postoperative adhesions and lack of tubular tissue in the porta hepatis area in subsequent surgery or liver transplantation. Creating a hepatoenterostomy outside of the porta hepatis should make it possible to avoid the problems. While the results of our experiment warrant some optimism, we will have to wait before this type of derivation operation is applicable in practice. Further research into this area is underway: anastomosis is attempted in an animal model.

\section{References}

1. Karrer FM, Lilly JR, Stewart BA, Hall RJ. Biliary atresia registry, 1976 to 1989. J Pediatr Surg 1990; 25 (10): 1076-1081.

2. Oh M, Hobeldin M, Chen T, Thomas DW, Atkinson JB. The Kasai Procedure in the Treatment of Biliary Atresia. J Pediatr Surg 1995; 30 (7): 1077-1081.

3. Mlakar B, Gadzijev EM, Ravnik D, Hribernik M. Congruence between the courses of the biliary ductal and the hepatic arterial systems. Eur J Morphol 2005; 42 (3): 135-141.

4. Ohi R. Surgery for biliary atresia. Liver 2001; 21 (3): 175-182.

5. Schneider BL, Mazariegos GV. Biliary atresia: A transplant perspective. Liver Transpl 2007; 13 (11): 1482-1495.
6. Sokol RJ. Biliary Atresia Screening: Why, When, and How? Pediatrics 2009; 123 (5): e951-e952.

7. Utterson EC, Shepherd RW, Sokol RJ, Bucuvalas J, Magee JC, McDiarmid SV et al. Biliary Atresia: Clinical Profiles, Risk Factors and Outcomes of 755 Patients Listed for Liver Transplantation. J Pediatr 2005; 147 (2): $180-185$.

8. Skandalakis JE et al. Surgical anatomy: The embryologic and anatomic basis of modern surgery. Athens; Paschalidis Medical Publications, 2004: 1007-1086.

9. McKiernan PJ, Baker AJ, Kelly DA. The frequency and outcome of biliary atresia in the UK and Ireland. Lancet 2000; 355 (9197): 25-29.

10. Endo M, Katsumata K, Yokoyama J, Morikawa Y, Ikawa H, Kamagata $\mathbf{S}$ et al. Extended dissection of the portahepatis and creation of an intususscepted ileocolic conduit for biliary atresia. J Pediatr Surg 1983; 18 (6): 784-793.

11. Ito T, Nagaya M, Ando H, Niinomi N, Iyomasa Y. Modified hepatic portal enterostomy for biliary atresia. Z Kinderchir 1984; 39 (4): 242-245.

12. Toyosaka A, Okamoto E, Okasora T, Nose K, Tomimoto Y, Seki Y. Extensive dissection at the porta hepatis for biliary atresia. J Pediatr Surg 1994; 29 (7): 896-899.

13. Ando H, Seo T, Ito F, Kaneko K, Watanabe Y, Harada $T$ et al. A new hepatic portoenterostomy with division of the ligamentum venosum for treatment of biliary atresia: a preliminary report. J Pediatr Surg 1997; 32 (11): 1552-1554.

14. Schweizer P, Kirschner HJ, Shittenhelm C. Anatomy of the porta hepatis as rational basis for the hepato-portoenterostomy. Eur J Pediatr Surg 1999; 9 (1): 13-18.

15. Chardot C, Carton M, Spire-Bendelac N, Le Pommelet C, Golmard $\mathbf{J}$, Reding R et al. Is the Kasai operation still indicated in children older than 3 month diagnosed with biliary atresia? J Pediat 2001; 138 (2): 224-228.

16. Hussein A, Wyatt J, Guthrie A, Stringer MD. Kasai portoenterostomy - new insights from hepatic morphology. J Pediatr Surg 2005; 40 (2): 322-326.

17. Altman RP, Chandra R, Lilly JR. Ongoing cirrhosis after successful porticoenterostomy in infants with biliary atresia. J Pediatr Surg 1975; 10 (5): 685-691.

18. Sokol RJ, Mack C, Narkewicz MR, Karrer FM. Pathogenesis and outcome of biliary atresia: current concepts. J Pediatr Gastroenterol Nutr 2003; 37 (1): 4-21. 